\title{
Lumen
}

Selected Proceedings from the Canadian Society for Eighteenth-Century Studies

\section{Les Amérindiens dans les Mémoires de la marquise de La Tour du Pin (1778-1815)}

\section{Servanne Woodward}

Volume 24, 2005

Indigenes and Exoticism

Indigènes et exotisme

URI : https://id.erudit.org/iderudit/1012173ar

DOI : https://doi.org/10.7202/1012173ar

Aller au sommaire du numéro

Éditeur(s)

Canadian Society for Eighteenth-Century Studies / Société canadienne d'étude du dix-huitième siècle

ISSN

1209-3696 (imprimé)

1927-8284 (numérique)

Découvrir la revue

Citer cet article

Woodward, S. (2005). Les Amérindiens dans les Mémoires de la marquise de La Tour du Pin (1778-1815). Lumen, 24, 35-48. https://doi.org/10.7202/1012173ar

Copyright (c) Canadian Society for Eighteenth-Century Studies / Sociéte canadienne d'étude du dix-huitième siècle, 2005
Ce document est protégé par la loi sur le droit d'auteur. L'utilisation des services d'Érudit (y compris la reproduction) est assujettie à sa politique d'utilisation que vous pouvez consulter en ligne.

https://apropos.erudit.org/fr/usagers/politique-dutilisation/ 


\section{Les Amérindiens dans les Mémoires de la marquise de La Tour du Pin (1778-1815)}

Lorsque nous recherchons la trace de l'histoire d'un peuple à travers les écrits (souvent autobiographiques) de leurs colonisateurs, nous rencontrons de nombreux écueils, et souvent de grandes déceptions. La littérature de voyage ne donne qu'une vision superficielle de ce peuple, et au mieux, ce sont la psychologie et les préoccupations de l'auteur qui nous sont révélées. Les «sauvages» font l'objet d'allusions rapides sans qu'il ne soit question d'une véritable solidarité humaine, entre autres parce qu'elle entraînerait peut-être l'addition embarrassante (sous tous rapports) d'éventuelles restitutions ou réparations. Il ne s'agit jamais de mettre en équivalence relative ni les peuples ni les cultures, et les Mémoires de la marquise de La Tour du Pin (1778-1815) ${ }^{1}$ ne font pas exception, d'autant plus que l'auteur (1770-1853) vient d'une caste qui a l'habitude de distinguer scrupuleusement entre les êtres humains selon leurs «quartiers» de noblesse ou l'ancienneté de leur «lignage» par rapport aux illustres familles françaises. Les Mémoires sont donc d'une banalité exemplaire dans la représentation des Amérindiens, parce qu'ils servent essentiellement à la gloire de l'autoportrait de l'auteur. Il faut noter, cependant, que la marquise exploite le mythe du "noble sauvage» pour établir sa distinction sociale par rapport aux autres colons, et «naturaliser» ainsi sa noblesse. Il s'agit pour elle de confirmer son rang, et de maintenir, voire de redéfinir les privilèges définissant sa «qualité». A ce propos, il faut noter que 1815 est la date de restauration pour la monarchie Bourbon, et c' est également la date à laquelle la marquise finit

1 Henriette-Lucy Dillon, marquise de la Tour du Pin, Mémoires de la marquise de la Tour $d u$ Pin. Journal d'une femme de cinquante ans (1778-1815) Suivi d'extraits de sa correspondance (1815-1846), présenté par son descendant le comte Christian de Liedekerke Beaufort, Paris, Mercure de France, 1979 et 1989, 611 p. 
ses Mémoires. Cette coïncidence invite à considérer que le retour de la monarchie cadre avec le but ultime du récit.

D'autre part, lorsque nous doublons la lecture littéraire de son texte par celle des documents historiques nous provenant des personnes fréquentées par la marquise, et dont les noms apparaissent dans les Mémoires, les Amérindiens deviennent de plus en plus centraux aux enjeux de l'autoportrait qui constitue le noyau de ces Mémoires. Harry Liebersohn relève le parallèle que les Français exilés entre 1789 et 1815 établissaient entre leur situation et celle des Amérindiens, à cause des «lois» qui expropriaient ces derniers de leur continent en maintenant une apparence de légitimité. ${ }^{2}$ Les Mémoires esquissent en filigrane une relation qui se confirme par les documents historiques, et qui révèle un certain degré de connaissance quant au programme d'expropriation qui les affecte. Le point de vue de la marquise correspond à cette perspective décrite par Liebersohn :

Titled Europeans had themselves been recent victims of popular land hunger [...]. They could readily see through the deceptions of written treaties for Indian lands and knew the hardship of being driven from one's home. To be sure, many titled Europeans clambered back to status and comfort despite the loss of legal privileges. $^{3}$

Les Européens ayant un titre de noblesse avaient été eux-mêmes les victimes récentes de l'avidité populaire pour la possession de terres [...]. Ils pouvaient saisir immédiatement les tromperies des traités écrits concernant les territoires Indiens et ils connaissaient les rigueurs endurées par les expropriés. Bien sûr, un grand nombre de nobles européens réussirent à retrouver un statut et leur confort en dépit de leur perte de privilège légal (Ma traduction).

C'est ainsi qu'ayant atteint un nouvel équilibre de confort, la marquise se situe à la convergence des valeurs aristocratiques, coloniales et amérindiennes. Le portrait de soi n'est pas détaché de la généalogie, ni de la «condition» de l'auteur qui s'aligne sur des courants d'idées pouvant faire partie d'un consensus commun.

Ce dernier est le thème récurrent de l'introduction de l'épisode américain. Quelques paragraphes d'introduction font référence aux Confes-

2 Aristocratic Encounters: European Travelers and North American Indians, Cambridge, Cambridge University Press, 1998, p. 2.

3 Aristocratic Encounters, op. cit., p. 3. 
sions de Rousseau, offrant ainsi une garantie de sincérité, et honorant le modèle littéraire canonique de l'écriture de soi. De la sorte, les Mémoires se présentent dans le cadre d'une attente créée par le genre des Confessions. De même, lorsqu'il s'agit ensuite de décrire l'aura séductrice de sa mère, nous restons dans le consensus: «les hommes l'adoraient, et les femmes n'en étaient pas jalouses, ${ }^{4} \gg$ ce qui a pour conséquence d'en faire une «dame du palais ${ }^{5} . »$ La singularité de la mère est de maintenir l'approbation générale au point de s'élever à la cour.

Immédiatement après, il est dit que la noblesse de la famille de la marquise peut rivaliser avec celle de la famille royale. En effet, son père a un équipage de chasse si brillant qu'il a de quoi contrarier le roi ${ }^{6}$. Par cette remarque, la marquise signale d'un coup sa puissance économique révolue et son privilège, qui lui perdure, non plus sous la forme du droit exclusif qu'avaient eu les nobles à pratiquer la chasse, mais par la mention de son engouement personnel pour cette activité. La chasse et la guerre se retrouvent d'ailleurs au centre des lois françaises définissant la noblesse et qui vont se communiquer au mythe du "noble sauvage» étudié récemment par Ter Ellingson ${ }^{7}$. Par conséquent, la marquise souligne le fait que la chasse et la guerre sont les activités des hommes qui constituent sa généalogie, et son commerce cordial avec les Amérindiens pourrait se justifier de leur activité essentielle de chasse et de guerre. De sa propre initiative, un chasseur guerrier nommé John lui tient lieu de garde du corps. Il est présenté l'arc à la main, accompagnant mystérieusement son équipage.

L'association entre l'Amérique et le père de la marquise est fortement soulignée: «[...] ]en Amérique où il faisait la guerre à la tête du premier bataillon de son régiment ${ }^{8}$.» La marquise apporte cette précision: «Le régiment de Dillon était entré au service de France en 1690, lorsque Jacques II eut perdu toute espérance de remonter sur le trône, après la bataille de la Boyne. Mon arrière grand-père, Arthur Dillon, le commandait ${ }^{9} . »$ Ainsi le rapport à l'Amérique se situe à l'origine des sphères d'action familiale - il a toujours été, et donc la destinée ou la destination

4 Mémoires, p. 38.

5 Mémoires, p. 39.

6 Mémoires, p. 43.

7 The Myth of the Noble Savage, Berkeley, University of California Press, 2001, p. 22-24.

8 Mémoires, p. 44.

9 Mémoires, p. 44. 
américaine de la Marquise est justifiée d'emblée. Mais également, la famille de la marquise a toujours partagé étroitement les hautes sphères du pouvoir. Le lien aux Amérindiens relève d'une longue familiarité entre les ancêtres de la marquise - de noblesse féodale - , et les autochtones d'Amérique du Nord - de la noblesse de l'Âge d'Or. Une noblesse (féodale) découle de l'autre (celle de l'Âge d'Or) et la marquise régénère son illustre condition dans sa proximité aux Amérindiens. Elle est «naturalisée» noble dans son accord amical ou sa conformité avec les Amérindiens et dans ses amitiés avec les familles dirigeantes de la ville d'Albany.

La marquise partage avec Marie-Antoinette la tentation aristocratique du siècle à apprécier et à assimiler les valeurs bourgeoises. Entre autres, elle sympathise avec le modèle révolutionnaire américain ${ }^{10}$. Elle s'inscrit dans l'apparition d'une noblesse nouvelle au Canada. Il se peut aussi que ce phénomène soit moins révolutionnaire que conforme à la pratique générale de l'anoblissement au début du XVII ${ }^{\mathrm{e}}$ siècle $^{11}$. Quoi qu'il en soit, l'appartenance de la marquise à la nouvelle noblesse canadienne ne peut que la confirmer dans l'ancienne. C'est dans cet esprit qu'elle adopte le monde de la ferme, comme avant elle le fondateur du mythe du «noble sauvage» selon Ellingson : il s'agit de Marc Lescarbot, un avocat qui a décrit les Amérindiens de 1'Est du Canada autour de $1609^{12}$. Le mélange de simplicité campagnarde avec le train de vie aristocratique établit la tonalité des Mémoires. Un principe d'hospitalité et d'entente régit les rapports des nouveaux venus avec les anciens habitants, ou entre les diverses catégories de la société.

Ainsi, l'amitié avec la jeune paysanne qui s'occupe d'elle lorsqu'elle est enfant a entre autres pour écho sa familiarité distante avec Judith, son esclave noire:

Tous les quinze jours, Judith lavait le linge des nègres, le sien et celui de la cuisine. Je lavais le mien, celui de mon mari et celui de M. de Chambeau, et je repassais le tout. Cette dernière partie de l'ouvrage était fort de mon goût. J'y excellais comme la meilleure repasseuse. Dans ma première jeunesse, avant mon mariage,

10 Rensläer fait venir un grand nombre de colons sur ces terres de l'Amérique, que Mme $\mathrm{d}^{\prime}$ Hénin considère dans son courrier à la marquise comme «le pays de la véritable liberté» (Mémoires, p. 237).

11 Ellingson, The Myth of the Noble Savage, op.cit., p. 32-33.

12 Ibid., p. 13. Voir Marc Lescarbot, Histoire de la Nouvelle France, Paris, Iean Milot, 1609. 
j'allais souvent à la lingerie, à Montfermeil, où, comme par une sorte de pressentiment, $\mathrm{j}^{\prime}$ avais appris à repasser ${ }^{13}$.

Mme de la Tour du Pin, habillée comme toutes ses voisines, se lève avec elles dès trois heures du matin, taillant un gigot à la hache. C'est ainsi qu'elle est surprise lors d'une visite inattendue de Monsieur de Talleyrand - visite coïncidant avec la chute de Robespierre et du tribunal révolutionnaire: "On ne peut embrocher un gigot avec plus de majesté $^{14}$, , s'exclame Talleyrand accompagné de Monsieur de Baumetz. Les rires de la marquise et de ses visiteurs rendent ce passage saillant, définissant pour l'épisode américain des Mémoires.

Le lignage guerrier de la marquise, sa jeunesse passée à la chasse, une similitude phonétique superficielle entre son activité et le nom de ses visiteurs (qu'elle invite finalement à partager les «beaux» «mets» qu'elle a «taillés»), la condamnation à mort et l'entaille charnelle de la marquise devenue bouchère, se mêlent et se font écho ${ }^{15}$. Il établit aussi la jonction particulière de la psychologie des Mémoires, entre une "simplicité» fermière, l'idéal rousseauiste de la Suisse - dont son imaginaire du citoyen - d'une part, et d'autre part, le milieu de la noblesse, ses valeurs, et ses responsabilités vis-à-vis des massacres qui viennent de la décimer. La marquise participe au geste révolutionnaire (le couperet) sur le mode noble (la hache guerrière ou sauvage de la guerre et de la chasse), mais dans un contexte bas et intime ou domestique (la cuisine, l'hygiène du coiffeur). L'effet projeté par cet épisode du gigot définit la marquise : elle est une cuisinière haussée au rang d'Amazone ou de Diane chasseresse, ou bien une Diane bouchère (à la fois sauvage et domestique, d'une iconographie royale et révolutionnaire) ou bien une Jeanne d'Arc " sauvage » au milieu des forêts d'Amérique qui aurait combiné son tablier avec la hache guerrière. Le mélange de cuisine (bassement utilitaire) et de guerre opère un mélange des genres.

Ce mélange est récurrent. Précédemment, lors de son voyage en mer, voyage lent et difficile, la marquise s'est coupé les cheveux par mesure d'hygiène, et cette coupe utile a convaincu les Bostoniens qu'elle venait tout juste d'échapper au supplice de la guillotine, symbole de guerre

\section{Mémoires, p. 278.}

14 Mémoires p. 242.

15 Il s'agit d'un processus d'unification comique (p. 138) ou de condensation (p. 70-72) telles que définies par Sigmund Freud dans Le mot d'esprit et sa relation à l'inconscient, traduction de Denis Messier, Paris, Gallimard, 1988. 
civile. Elle participe au geste du bourreau révolutionnaire sur le mode noble, et cet accompagnement reste ambigu, comme s'il s'agissait de trancher, de se détacher d'un état de noblesse antérieur. La marquise devient une figure composite héroïcomique, associant des modes incongrus, à la fois révolutionnaires, nobles et sauvages.

De cette manière, la marquise partage un statut de «survivante» avec les «sauvages» Amérindiens. Nous sommes d'abord frappés que les Amérindiens soient présentés comme des êtres obsolètes, en voie de disparition, voués donc de façon incontournable à s'évanouir de la terre où ils ne resteraient que par le nom:

Ceux-là étaient les derniers survivants de la nation des Mohawks, dont le territoire a été acheté ou pris par les Américains depuis la paix. Les Onondagas, établis près du lac Champlain, vendaient aussi leurs forêts et se dispersaient également à cette époque. Il en venait quelques-uns de temps à autre. Je fus un peu surprise, je l'avoue, quand je rencontrai pour la première fois un homme et une femme tout nus se promenant tranquillement sur la route, sans que personne songeât à le trouver singulier. Mais je m'y accoutumai bientôt, et lorsque je fus établie à la ferme, j'en voyais presque tous les jours pendant l'été ${ }^{16}$.

Les Onondagues font des mocassins dont la description détaillée montre assez qu'il s'agit de chaussures étrangères, mais dont la marquise désire faire l'achat. Le seul fait que le mot mocassin soit souligné dans le texte désigne l'objet comme externe au vocabulaire de l'auditoire français présumé des mémoires. Mme de la Tour du Pin se prête aux coutumes de sa nouvelle communauté par courtoisie. Les épisodes Onondagues sont preuve de la parfaite implantation de Mme de la Tour du Pin en Amérique. Elle semble même très informée au sujet des limites des territoires et de leur mode d'acquisition. Ainsi lorsque la marquise parle de la visite du duc de Liancourt: «Il arrivait des nouveaux établissements formés depuis la guerre de l'indépendance sur les bords de la Mohawk et dans le territoire cédé par la nation des Onéidas ${ }^{17}$.» Tout semble pris dans une dynamique d'import, d'appropriation et d'emprunt. Finalement, mieux que «dans leurs chaussures,» la marquise est à sa place «à leur place» et bien implantée. L'entente avec les Iroquois témoigne de sa «naturalisation» parfaite au territoire amérindien. Il y va de la légitimité de son immigration à Albany : cette intégration immédiate confirme qu'elle est peut-être plus compatible avec les premiers habitants, du fait

16 Mémoires, p. 245.

17 Mémoires, p. 263. 
de leur noblesse commune, ou des antécédents américains des aïeux de la marquise, antérieurs à l'arrivée massive des colons d'Albany.

Ainsi ses rapports personnels sont tout à la fois cordiaux et marqués par sa provenance externe, du vieux monde. Les personnes secondaires - dont les Amérindiens - éclairent divers aspects de la narratrice mais surtout elles se classent en groupes distincts par leur accueil. Les gens qui ont un lien d'amitié immédiat attestent de la bonté de caractère ou de la bonne réputation familiale de la marquise. Les créoles se rappellent du père de la marquise de façon avantageuse, comme Black, la chienne du capitaine jamaïcain, s'échappe du bateau pour les rejoindre, et comme les esclaves réclament la marquise pour propriétaire.

Mais il s'agit là des êtres domestiques et non «sauvages» cependant, et c'est là que se joue la spécificité des «squaws" en tant qu'êtres sauvages. Ils font partie d'un commerce très limité. Il s'agit moins d'un rapport «commercial» qui reste le propre des colons paysans ou bourgeois. Il s'agit plutôt d'échanges de bons procédés et d'amitiés, dans la gratuité du geste authentiquement noble. Leur marginalité rappelle en quelque sorte celle de la marquise. C'est sur le plan de l'appréciation des valeurs que se confirme l'état de noblesse, antithétique au travail et à l'échange marchand. Les mocassins très décorés (donc chargés d'une valeur esthétique non utilitaire) et les paniers perméables à l'eau "coûtent» fort peu à la marquise. Elle fait cadeau d'objets de peu de valeur, et de quantités dérisoires de beurre par rapport à la qualité et au travail du panier, alors que les mocassins coûtent fort cher aux autres habitants n'ayant pas d'affinité particulière avec les nobles sauvages: «le prix de ces objets est quelquefois assez élevé, quand ils sont brodés avec de l'écorce teinte ou avec des piquants de porcs-épics ${ }^{18} . »$ Mais en sa qualité d'aristocrate, immédiatement reconnue par les Amérindiens, la marquise est exemptée de la sphère commerciale. «Squaw John» lui fait cadeau d'un fouet ${ }^{19}$. Elle reçoit des cuillères et des jattes de bois

18 Mémoires, p. 245.

19 D'autres passages montrent que la marquise conserve ses distances. Evidemment, malgré leur christianisation, les Amérindiens restent externes comme dans le paragraphe suivant, où la marquise converse avec «squaw John.» Le baptême (John semble indiquer qu'il y a eu baptême) ne leur enlève pas l'épithète "squaw». Ils sont également suspects de vouloir enlever les enfants: parce que son fils risque de tomber de cheval, se mêle aux esclaves dans les champs, et suit les Amérindiens, la marquise s'inquiète et le confie à une dame sans enfants (Mémoires, p. 257-58). C'est encore leur «costume bizarre» qui effraie M. de Novion qui est dissuadé de devenir fermier dans «un pays où l'on était exposé à de semblables rencontres» (Mémoires, p. 257). 
découpées au tomahawk pour sa laiterie. Comme dans le cas de la hache du gigot, l'arme guerrière est utilisée à une tâche qui en devient moins domestique. La hache et le tomahawk marquent un principe de résonance entre deux noblesses, celle de la féodalité et celle de l'Âge d'Or.

Les Onondagues confirment la noblesse de la Marquise:

Cependant, ces sauvages à peine familiarisés avec quelques mots d'anglais, qui passaient leur été à courir de ferme en ferme, étaient aussi sensibles aux bons procédés, à une réception amicale, que l'aurait été un seigneur de la cour. Ils avaient bientôt compris que nous n'appartenions pas à la même classe que les fermiers nos voisins. Aussi disaient-ils en parlant de moi: Mrs Latour... from the old country... great lady ... very good to poor squaw. Ce mot de squaw signifie sauvage. Il qualifie indifféremment tout être ou tout objet provenant des pays où la civilisation européenne n'a pas encore pénétré. Ainsi il s'applique aux oiseaux de passage: squaw pigeon, squaw turkey; aux objets apportés par les sauvages: squaw baskett, etc., etc. ${ }^{20}$

C'est apparemment la «nature» même qui reconnaît la noblesse de la marquise. Ainsi tout ce qui est «squaw» participe à un effet de la poussée du printemps dans le passage suivant:

A propos du printemps, il est intéressant de rapporter avec quelle promptitude il arrivait dans ces parages. La latitude, $43^{\circ}$, se faisait sentir alors et reprenait tout son empire. Le vent du nord-ouest, après avoir régné tout l'hiver, cessa brusquement [...] Aussi, en moins d'une semaine, les prés verdissaient, se couvraient de fleurs et une innombrable variété de plantes de toute espèce, inconnues en Europe, remplissaient les bois. Les sauvages, qui n'avaient pas paru de tout $l^{\prime}$ hiver, recommencèrent à visiter les fermes $[\ldots]^{21}$.

L'entente, la conformité, l'harmonie s'opèrent avec une distance, avec discernement. Les Amérindiens produisent des objets sauvages comme la nature produit des pigeons, et cependant ils sont pauvres, c'est-à-dire réduits à l'austérité - nus - et dépendant de la générosité de la marquise. La marquise donne, peut-être plus généreusement que d'autres colons.

La différence de la marquise par rapport aux fermiers est discernée par les Amérindiens. Sa conformité apparente aux autres colons est

20 Mémoires, p. 255-256.

21 Mémoires, p. 253-254. 
superficielle. La marquise porte délibérément ses mocassins et ses jupes de laine paysanne: «La jupe de laine bleue et noire rayée, la petite camisole de toile de coton rembrunie, le mouchoir de couleur, les cheveux séparés comme on les porte maintenant et relevés avec un peigne $[\ldots]^{22}$ ». Cet accoutrement donne un mouvement de méprise au duc de Liancourt qui vient lui rendre visite: «avait-il pris au sérieux ma jupe de laine et ma camisole de toile ${ }^{23}$ ? » Après s'être changée, pour porter une robe de grand luxe, elle exige qu'il se change lui-même: «[...] avec ses vêtements couverts de boue, de poussière, déchirés en plusieurs endroits, il avait l'air d'un naufragé échappé aux pirates, et personne n'aurait pu se douter que sous cet accoutrement bizarre se cachait un premier gentilhomme de la Chambre ${ }^{24}$.» Ce n'est pas le vêtement immédiat qui signifie la classe, mais l'accessibilité à une garde-robe noble, propre et intacte. La nudité des Amérindiens situe leur noblesse hors de la civilisation, sur le mode archaïque. Ils sont non seulement d'un autre monde, mais $d^{\prime} u n$ autre temps, hors de la société perfectible peut-être à cause de leur appartenance à l'Âge d'Or comme temps révolu de perfection, sujet à une nostalgie du passé plutôt qu'à la marche du présent. Plus encore que leur noblesse, ce qui les rendrait antithétiques aux colons bourgeois, ce serait donc leur appartenance à un monde antique et révolu.

La porcherie et la laiterie de la marquise appartiennent au mode de la perfectibilité, parce qu'elles sont finalement exemplaires. Elles sont nobles, prises dans l'esthétique ambiguë de l'utilitaire et du luxe. Il s'agit encore d'un Trianon: «a noble hog sty ${ }^{25}$ !» L'élégance fermière n'est pas sans rappeler la bergerie de Marie-Antoinette. Cette nature est entièrement transformée, non seulement domestiquée mais peut-être dans la sphère de la pure fiction ludique, un objet de culture. Le «Hameau de la Reine» à Versailles prétendait à ce même équilibre. Ce lieu de retraite, permettant la production de produits laitiers, contenait aussi une grotte artificielle évoquant la perfectibilité de l'homme à travers les âges et dans laquelle la reine se reposait lors de la marche sur Versailles le 5 octobre 1789, nous rappelle Patrice Higonnet ${ }^{26}$. Quant aux Amérindiens,

24 Mémoires, p. 264.

25 Mémoires, p. 263.

26 «Mique, the Architect of Royal Intimacy» dans Michael Conan (éd.), Bourgeois and Aristocratic Cultural Encounters in Garden Art 1550-1850, Washington D.C., Dumbarton Oaks, 2002, 25-41, p. 34, n. 18 et p. 35. 
comme «squaw» ils proviennent tout simplement de la nature sauvage, c'est-à-dire selon la définition de la marquise, ce qui n'a pas été transformé par la culture européenne. Les «derniers survivants» amérindiens semblent appelés à se «disperser» dans la nature: «Les Onondagas, établis près du lac Champlain, vendaient aussi leurs forêts et se dispersaient également à cette époque ${ }^{27}$.» Nous connaissons la suite de cette catégorisation des Amérindiens en personnes qui se «dispersent», s'effacent de façon accommodante dans le paysage pour nous céder leur place. Plus troublante encore est cette catégorisation des Amérindiens en "survivants" comme s'ils étaient en sursis par rapport à une extinction imparable.

Toute la sensibilité romantique proviendrait du choc de la Révolution et du sentiment d'appartenir à un monde défunt, dont les supériorités du coeur - courage guerrier ou charité - seraient supplantées par une société bourgeoise, donc mercantile. Ce serait entre autres l'un des aspects de la mélancolie des Mémoires d'outre tombe. Ainsi s'explique peut-être l'un des aspects de l'identité que la marquise perçoit entre son monde et celui des Amérindiens. Encore semble-t-elle entièrement positive et optimiste, dans la veine de l'appartenance au XVIII ${ }^{e}$ siècle des Lumières, résolûment tournée vers la construction de l'avenir, l'aspect durable de l'action, et la participation au gouvernement. Pour son compte, la marquise s'assimile aux familles dirigeantes, à la population puissante et majoritaire, plutôt que de considérer l'extinction. Sans doute prévoit-elle l'extermination ou l'assimilation des Amérindiens sous l'angle du progrès ou de la marche inévitable des choses vers la «civilisation.» Ce fut le point de vue que partageaient Lescargot et Louis Hennepin ${ }^{28}$. Alors que la marquise a fait preuve de fermeté en libérant les esclaves qu'elle employait avant de repartir pour la France, elle ne semble pas avoir oeuvré pour les Amérindiens. Il se peut que les configurations du pouvoir ne lui en aient pas donné l'occasion, alors que les lois permettaient l'émancipation d'esclaves. Il se peut qu'elle ait, volontairement ou non, consciemment ou non, participé à leur infortune.

Car à leur sujet, la marquise en sait sans doute beaucoup plus long qu'il n'y paraît, puisqu'elle répète suffisamment souvent le nom de Schuyler parmi le cercle de notables qu'elle fréquente assidûment. Or la famille Schuyler avait fort à faire avec les Amérindiens :

27 Mémoires, p. 245.

28 Voir Ellingson, The Myth of the Noble Savage, op.cit., p. 27, p. 31-32; p. 62. 
On September 15, 1795, a group of Oneidas, armed with state-provided power of attorney, signed an accord with four New York State Indian commissioners - Philip Schuyler, David Brooks, John Cantine, and John Richardson whereby the Indian «sold» to the state more than 100,000 acres of their choicest tribal lands in what is today Oneida and Madison counties, for a one-time payment of $\$ 2,952$ and an annual payment of $\$ 2,952$. Going against tribal sentiments expressed by Good Peter before his death and other Oneida sachems not to cede any more land, these Oneidas sold a portion of their land around Oneida Lake, the south and east sides, but reserved the Indian's right to lake access and fish and retained ownership rights to half-mile sections along the north shore. Although this state-Oneida accord has been written about before, the full background of this dispossession, including the major player in this high jinx, has never been disclosed.

At the center of these events was Philip Schuyler, the commanding general of Oneida troops during the American Revolution [...] Schuyler, through his actions and those of his cronies, violated the federal Trade and Intercourse Acts of 1790 and 1793 [...] Nearly every political decision and treaty negotiation involving the Oneidas between 1785 and 1798 had Philip Schuyler's personal or family stamp of approval ${ }^{29}$.

Le 15 septembre 1795, un groupe d'Oneidas, armés du pouvoir juridique d'état, ont signé un accord avec les commis aux Affaires Indiennes de l'État de New York - Philip Schuyler, David Brooks, John Cantine, et John Richardson - par lequel les indiens ont «vendu» à l'État plus de 100,000 acres de leurs meilleures terres tribales de ce qui est connu aujourd'hui comme les comtés d'Oneida et de Madison, pour un paiement unique de \$2,952 et une annuité de $\$ 2,952$. Allant contre les sentiments tribaux de Good Peter avant son décès et d'autres sachems Oneida de ne pas céder plus de terrain, ces Oneidas ont vendu une portion de leur terre autour du lac Oneida, au sud et à l'est, mais réservé les droits indiens d'accès au poisson et conservé la propriété d'une section d'un demi mile le long de la berge nord. Bien que cet accord d'état Oneida ait été l'objet de commentaires écrits, la toile de fond de cette perte de terre, y compris l'agent principal de cette extorsion, n'a jamais été exposée.

Au centre des événements se trouvait Philip Schuyler, le général commandant les troupes Oneida pendant la Révolution américaine [...]. Schuyler, à travers son action et celle de ses acolytes, violait les lois de commerce et

29 Laurence M. Hauptman, "Command Performance Philip Schuyler and the New York State-Oneida "Treaty" of 1795 ", dans The Oneida Indian Journey from New York to Wisconsin 1784-1860, Laurence H. Hauptman et L. Gordon McLester (édit.), Madison, The University of Wisconsin Press, 1999, 38-50, p. 38-39. 
d'interrelation de 1790 et 1793 [...] Pratiquement toute décision politique et négociation de traité impliquant les Oneidas entre 1785 et 1798 portait le tampon d'approbation de Philip Schuyler ou de sa famille (ma traduction).

Hauptman cite les beaux-fils de Schuyler: Alexander Hamilton, secrétaire trésorier, et le très riche Stephen Van Rensselaer. Schuyler avait gagné la confiance des Oneida en commandant leurs troupes pendant la Révolution. En tant que sénateur, cette confiance lui permit d'aller à l'encontre des lois fédérales pour s'enrichir, lui et sa famille, et s'attribuer la capitale, Albany, en tant que ville sous sa possession personnelle. Son point de vue était le suivant:

[...] for as our settlements approach their country, they must from the scarcity of game, which that approach will induce to, retire further back, and dispose of their lands $[\ldots]$ they [will] dwindle comparatively to nothing, as will savages have done, who reside in the vicinity of civilized people and leave us the country without the expense, trifling as it may be of a purchase ${ }^{30}$.

[...] car lorsque nos colonies s'approchent de leur pays, ils doivent, à cause de la rareté du gibier, que cette approche provoquera, se retirer plus loin, comme les sauvages ont toujours fait, lorsqu'ils résident dans le voisinage des gens civilisés, et ils nous laissent le pays sans nous causer de dépense, aussi ridicule soit-elle (ma traduction).

Ainsi, la famille Schuyler recrutait activement des colons parmi les immigrés de la Révolution française: « [...] Mme d'Hénin, notre tante, tout en regrettant que nous n'eussions pas été la rejoindre en Angleterre, nous envoyait des lettres d'une Américaine de ses amies, Mme Church, nous recommandant à sa fille en résidence à Albany. Mme Church était fille du général Schuyler, qui s'était créé une grande réputation dans la guerre de l'Indépendance ${ }^{31}$.» Et plus loin, «nous reçûmes, à notre arrivée à Boston, des lettres très pressantes du général Schuyler par lesquelles il nous engageait à nous rendre sans délai à Albany où, assurait-il, nous

30 Hauptman, «Command...», p. 39, citant Philip Schuyler, «Thoughts Respecting Peace in the Indian Country, » July 29 1783, Philip Schuyler MSS, Notes of August 61795 and August 8 1795, MR7, New York Public Library.

31 Mémoires, p. 230-31. 
trouverions aisément à nous établir. Il nous offrait dans ce but tout son appui $^{32}$.» De cette façon, le zèle que le général Schuyler déploie pour reconstruire Albany après qu'une révolte d'esclaves ait réduit la ville en cendres devient moins altruiste que ne le présente la marquise: «Les familles Renslaër et Schuyler firent des merveilles de charité éclairée et donnèrent l'exemple de l'activité à réparer le désastre ${ }^{33}$.» Enfin entre les Schuyler, les Renslaër et la marquise, il n'est question que d'amitié, d'invitation à dîner et même du don d'un cheval pour écraser les pommes afin de produire du cidre ${ }^{34}$. Les rapports sur les ventes de terrain viennent tout naturellement lors d'un commentaire à propos de la visite du duc de Liancourt: «Il arrivait des nouveaux établissements formés depuis la guerre de l'Indépendance sur les bords de la Mohawk et dans le territoire cédé par la nation des Onéidas. M. De Talleyrand lui avait remis des lettres pour les Schuyler et les Renslaër ${ }^{35} .{ }^{2} \mathrm{La}$ marquise n'est plus simplement un pion dans l'échiquier des projets Schuyler et Renslaër, elle est un agent actif du processus d'expropriation des Amérindiens. L'amitié attentive des Amérindiens est alors à relire dans ce contexte beaucoup moins idyllique que dans le cadre d'une noblesse partagée. Les grandes démonstrations d'amitié et l'attention extrême des degrés de politesse à observer de part et d'autre font véritablement partie d'un jeu diplomatique dont l'enjeu est la survie des Amérindiens et les terres que les colons désirent s'approprier. De secondaires, les Amérindiens deviennent de plus en plus centraux à l'épisode américain des Mémoires qui ne constitue qu'un chapitre de la vie de la marquise.

Et finalement le statut aristocratique de la marquise se mesure à sa capacité d'oeuvrer politiquement afin de rester au coeur de la communauté gouvernante, en tant que membre actif de la haute société. Ses divers déguisements ne doivent pas masquer l'essentiel, son appartenance à la caste gouvernante. Mais cet essentiel reste étrangement occulté, compréhensible finalement pour de peu nombreux lecteurs, ceux notamment qui sont au fait des jeux de pouvoir entre les Reinsler et les Six Nations, c'est-à-dire aux dirigeants et aux initiés informés, qui seuls ont les clés de lecture adéquates pour déchiffrer le train de vie de la marquise en Amérique. L'auditoire prévu par les Mémoires 
est donc manifestement la noblesse française intégrée aux instances dirigeantes. C'est ainsi que la marquise prévoyait ou constatait avec optimisme la survivance post-révolutionnaire des nobles français autour de 1815.

SERVANNE WOODWARD

Université Western Ontario 